\title{
Low temperature aging behaviour of transformation temperatures in some Cu-based and NiTi SMA
}

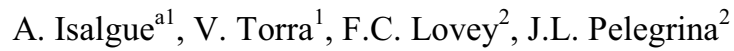 \\ ${ }^{1}$ CIRG, Departament Física Aplicada UPC, Campus Nord, B4, C. Jordi Girona 31, Barcelona 08034, SPAIN \\ ${ }^{2}$ División Metales, Centro Atómico Bariloche e Instituto Balseiro, San Carlos de Bariloche 8400, ARGENTINA
}

\begin{abstract}
Effects of aging at intermediate temperatures (near $400^{\circ} \mathrm{C}$ ) in SMA have been found to produce precipitation, which affects the transformation behaviour. In this work, we observe some dependences (recoverable or not) of transformation temperature with aging at temperatures near or under $100^{\circ} \mathrm{C}$ in beta phase. Two copper-based SMA alloys have been analyzed, CuZnAl and CuAlBe. Also, some analysis has been done on pseudo-elastic NiTi. By aging at temperatures near $100^{\circ} \mathrm{C}$, the two copper alloys show changes in transformation temperatures, which can be related univocally to the changes in the temperature at which the alloy is kept. In both alloys, by staying long time at a given temperature, an asymptotic value of the transformation temperature is reached, and further coming to the same temperature produces the transformation temperature to evolve to that asymptotic value. The maximal amplitude of the changes is about $16 \%$ of the amplitude of the change in aging temperature. The changes are much faster in $\mathrm{CuZnAl}$ than in $\mathrm{CuAlBe}$, and the sign of the changes is opposite in $\mathrm{CuAlBe}$ respect to that in CuZnAl. The process can be well modelled by a system of differential equations with one or two terms, giving a "tracking" of the transformation temperature respect the temperature at which the alloy is kept. The activation energies for the time evolution suggest atomic-order related processes as cause for the transformation temperature changes. For NiTi, the evolution is much slower, and seems to be monotonic with time, suggesting a precipitation-related mechanism as the origin of the transformation temperature changes, even at temperatures near $100^{\circ} \mathrm{C}$. From the data, some conclusions may be drawn concerning long-time effects on the applications of the alloys
\end{abstract}

\section{Introduction}

The martensitic transformation between metastable phases is the origin of the shape memory effects [1-3]. The applications of the shape memory material for a small number of on-off actions, needs only a relative reliability. Applications of shape memory alloys (SMA) as smart materials require a long time guaranteed behaviour. This supposes a fatigue life in accordance with the application, and a reproducible hysteretic behaviour. The mechanical properties of the SMA in the pseudo-elastic regime [1] make them attractive for damping devices, for instance in civil engineering [2-4]. The ability to support large, recoverable strains at moderate stress levels, and the presence of a considerable hysteresis are important parameters. In damping, the thermo-elasticity, the hysteresis, the maximum recoverable strain, and the Clausius-Clapeyron equation (relating the critical transformation stress with the room temperature) are the most relevant macroscopic properties. Also, careful attention is necessary to avoid perturbative effects related with diffusion.

When the SMAs are subjected to working cycles, some evolutive dependencies can appear [5]. The evolution of properties may have a mechanical origin, i.e., the classical creation and movement of dislocations, but may also have a thermal cause, i.e., diffusive effects as atomic order changes and vacancy concentration evolution, or even precipitation at intermediate temperatures. The alloys are usually subjected to quenching to preserve the high temperature or parent phase, avoiding precipitation of equilibrium phases in the cooling process. Then, the parent phase is considered a metastable one at room temperature. It has been observed in $\mathrm{Cu}$ based alloys that the quenching temperature has a strong influence on the transformation temperature, when the quenching is from intermediate temperatures, because of atomic ordering depending on temperature [6]. For the case of $\mathrm{NiTi}$, it has been reported that aging at temperatures from $713 \mathrm{~K}$ to $823 \mathrm{~K}$ has a strong influence on transformation temperatures, because of a precipitation-dissolution process [7].

\footnotetext{
ae-mail: antonio.isalgue@.upc.edu
} 
In fact, the time dependence of properties requires an evaluation to guarantee the behaviour [8]. In SMAs they are slow processes at moderate temperatures [5], but each possible application needs a detailed consideration of requirements the material has to fulfil, and the time-scales associated with the application requires an explicit consideration. For instance, damping of earthquakes might have a waiting time of many years or decades, while satellite launching needs only reliability for a few days, and medical applications might need some weeks or months. In this work, results of the low temperature aging effects on transformation temperature for two $\mathrm{Cu}$-based alloys $(\mathrm{CuZnAl}$ and $\mathrm{CuAlBe})$, and for NiTi are compared.

\section{Experimental}

We used CuZnAl single crystals of composition $\mathrm{Cu}-14.6$ at.\% Zn-16.2 at.\% Al, with electronic concentration 1.48 e/a. Samples of $2 \mathrm{~cm}$ length and near $0.5 \mathrm{~mm}^{2}$ cross section were used. Also, polycrystalline CuAlBe from Trefimetaux and Nimesis (France), with composition Al $11.8 \mathrm{wgt} \%$, Be $0.5 \mathrm{wgt} \%$, Cu to balance, was used. The $\mathrm{Cu}$-based alloys were subjected to betatisation at $1123 \mathrm{~K}$ for 10 minutes and water quench. The $\mathrm{CuAlBe}$ alloy in the as-furnished state (extruded wire) contained beta but also some alpha phase, and there were some scarce plates of martensite in the beta grains.

NiTi wire of $0.5 \mathrm{~mm}$ diameter (55.9 wgt $\%$ of $\mathrm{Ni}$ ) furnished in super-elastic form, from Special Metals (New Hartford, USA) and later SAES-getters, has also been used. The NiTi wires were used as furnished.

Electrical resistance measurements were done with a computer controlled and programmed temperature, 4-point technique, providing more than 4 significant figures in electrical resistance. The computerized device, controlling the sample temperature as described in [9], permits cooling and heating to determine the Ms value by electrical resistance measurements. The cycles were done at relatively low speeds, in order to determine properly the temperature (near four hours per cycle).

To characterize the phases present in NiTi alloy, An X-ray diffractometer INEL was used, with Cu tube, and graphite monochromator (using $\mathrm{K}_{\alpha 1}, \lambda=0.15406 \mathrm{~nm}$ ), with detector CPS 120 (5644 channels). For the temperature control we used an Oxford Cryosystems, 700 series cryo-stream plus. At room temperature (293 K), only cubic beta phase was detected by X-ray diffraction, suggesting that any possible precipitates in the superelastic wire are rather small and/or scarce.

\section{Results and discussion}

To determine quantitatively the evolution of the transformation temperature when the sample is kept in parent phase, several samples were used. $\mathrm{CuZnAl}$ single crystal and some CuAlBe polycrystalline samples were mainly used. Some parallel measurements on NiTi alloy have been also done, showing a similar general behaviour even though harder to detect because of larger hysteresis and temperature domains involved [10].

For the $\mathrm{Cu}$-based alloys $\mathrm{CuZnAl}$ and $\mathrm{CuAlBe}$, it has been observed that, for cycled samples, and after long time in parent (beta) phase at a given temperature $T$, and avoiding the coexistence of phases and dislocation creation effects, the transformation temperature Ms converges to a well-defined value dependent only on the temperature $T$ [11]. When the material undergoes a step like change of temperature the electrical resistance varies quickly via the classical phonon contribution (nearly linear dependence of resistance with temperature), as expected, but it only reaches the end value at constant temperature (the "steady" or "equilibrium" state) in an exponential way, with relatively large time constants near room temperature, from near $1 \mathrm{~h}$ at $373 \mathrm{~K}$ to about a year at $293 \mathrm{~K}$ in CuZnAl with electronic concentration 1.48 e/a [9]. A similar effect has been reported at higher temperatures in some alloys affected by diffusive atomic ordering processes [12]. The amplitude of the exponential variation of resistance remains near $5 \%$ of the phonon contribution. Long time after the quench, the $\mathrm{Cu}$-based alloys approach to the steady ("equilibrium") state is described satisfactorily by means of exponential decay terms $[5,11]$. The time constants $\left(\tau^{(i)}, i=1,2, \ldots\right)$ relate activated processes since they depend strongly on the temperature $T$, and these time constants can be written as:

$$
\tau^{\prime}=\operatorname{xp}\left(A_{i}+\frac{3_{i}}{T}\right)
$$

As the electrical resistance versus time follows a similar behaviour to the experimental evolution of Ms, this suggests the introduction of hidden internal "order" temperatures $T^{(i)}(i=1,2, \ldots)$ in the sample $[11,13]$, tracking the external or room temperature $T(t)$ via independent differential equations, as: 


$$
\frac{d T^{(i)}(t)}{d t}=-\frac{T^{(i)}(t)-{ }^{-}(t)}{\tau^{(i)}}
$$

And then the calculated time dependent Ms values are deduced from the $T^{(i)}(t)$ (the hidden order temperatures) starting in a steady state at external temperature $T^{\text {ref }}\left(\right.$ with $\mathrm{Ms}=\mathrm{Ms}\left(T^{\text {ref }}\right)$ ) by:

$$
\operatorname{Ms}(t)=\operatorname{Ms}\left(T^{\mathrm{ref}}\right)+\sum a_{i}\left(T^{(i)}(t)-T^{\mathrm{ref}}\right)
$$

Using a classical Runge-Kutta algorithm and starting in a steady state or in a known state the differential equations can be solved and the time dependent values of Ms can be calculated if the temperature profile $T(t)$ is known. The previous expressions allow to obtain the Ms changes induced from winter and summer temperatures (seasonal) smoothed by the tracking action in the differential equations.

\subsection{CuZnAl alloy}

The figure 1 shows the experimental temperature profile $\mathrm{T}(\mathrm{t})$, the measured transformation temperatures Ms (circles), and the computed Ms during 2 years for a CuZnAl single crystal, using two terms in the expression (3), with parameters $\mathrm{a}_{1}=-0.105 ; \quad \tau^{1}=\exp (-29.30+13630 / \mathrm{T}) ; \quad \mathrm{a}_{2}=-0.067 ; \quad \tau^{2}=\exp (-16.93+10330 / \mathrm{T}) \quad(\mathrm{T}$ in $\mathrm{K}$ and $\tau$ in s).

In a similar $\mathrm{CuZnAl}$ alloy, the temperature activated processes have been associated to an atomic reordering visualized by careful X-ray diffraction study [14]. In that case, and due to limited temperatures and times, only one term in equation (3) was fitted.

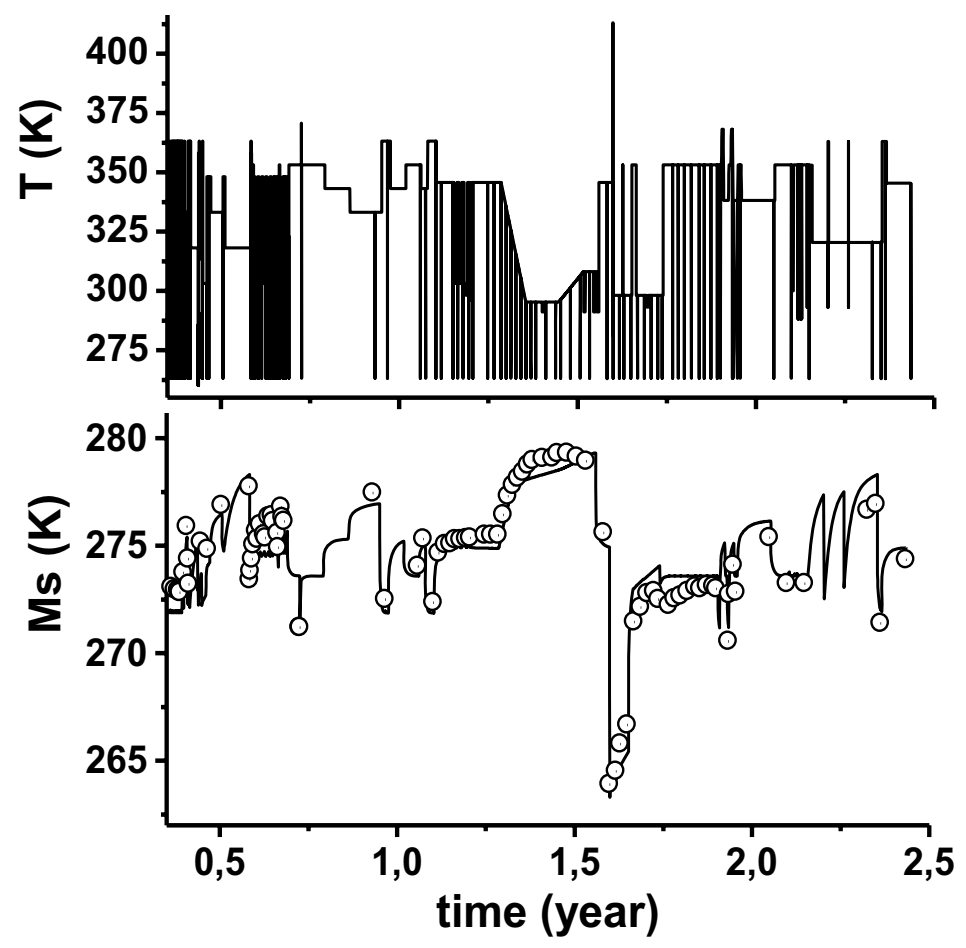

Fig. 1 Transformation temperature $\mathrm{Ms}(\mathrm{T}, \mathrm{t})$ for $\mathrm{CuZnAl}$ single crystal (1.48 e/a). Upper part: temperature profile. Lower part: Circles: experimental Ms; Continuous line: computed Ms evolution as indicated in the text.

\subsection{CuAlBe alloy}

For the CuAlBe polycrystal, long time after the quench (i.e., the zone after the vertical arrow in fig. 2), a good approach to the Ms dependence on temperature and time could be obtained with only one time constant (one 
term in expression 3), with an "a" coefficient near +0.13 , and a time constant $\tau$ as function of temperature ( $\mathrm{T}$ in $\mathrm{K}$ and $\tau$ in $\mathrm{s})$ :

$$
\ln \tau=-3.47+5740 / \mathrm{T}
$$

It has to be noted that, although the behaviour is analogue to that of $\mathrm{CuZnAl}$, the time constants near room temperature are much larger for $\mathrm{CuAlBe}$, the amplitude of the changes in $\mathrm{Ms}$ are somewhat lower for $\mathrm{CuAlBe}$, and the sign of the change is opposite in $\mathrm{CuAlBe}$ respect to $\mathrm{CuZnAl}$, i.e., if one increases the room temperature from a "steady" state, for $\mathrm{CuZnAl}$, the Ms tends to decrease, while in CuAlBe tends to increase, producing that in CuZnAl both Clausius-Clapeyron and diffusive effects ask for more stress to transform a sample if temperature is increased, but in $\mathrm{CuAlBe}$, the diffusive actions result opposite to the Clausius-Clapeyron relationship between the temperature changes and the changes in stress to transform a sample, smoothing the concomitant effects, the dependence of stress to transform with temperature and the transformation temperature changing with time due to diffusive effects.

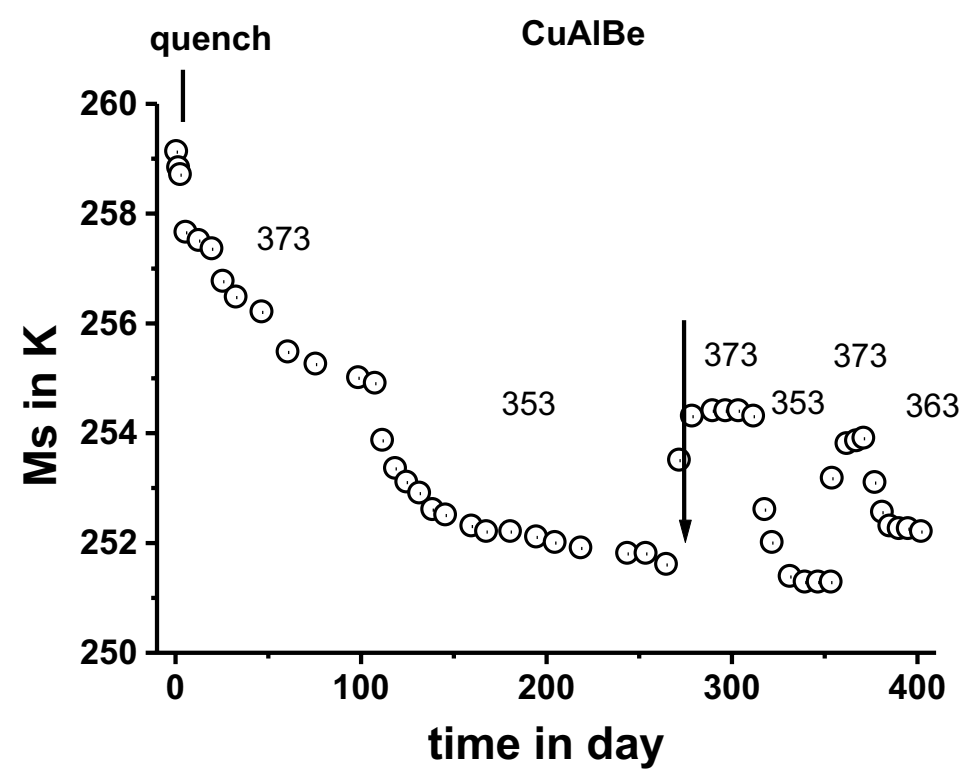

Fig. 2 Experimental transformation temperature $\mathrm{Ms}(\mathrm{T}, \mathrm{t})$ for $\mathrm{CuAlBe}$ polycrystal (circles). The numbers over the circles indicate the temperature at which the sample was kept in K.

\subsection{NiTi alloy}

The figure 3 shows the results obtained with samples of NiTi pseudo elastic wire. The results were obtained by decreasing the temperature smoothly to detect a phase transition, to $255 \mathrm{~K}$ or to $275 \mathrm{~K}$. X-ray measurements showed that at zero stress, only beta phase was detected at $293 \mathrm{~K}$, the martensite phase was detected at $148 \mathrm{~K}$, but only R-phase was detected at temperatures as low as $188 \mathrm{~K}$. Then, the electrical resistance was used to monitor the R-phase transformation temperature, Rs. By maintaining the sample at $373 \mathrm{~K}$, a smooth increase of Rs was observed during near a year (squares in fig. 3). The time dependence of Rs [15, 16] suggests a time constant of more than 2 months at $373 \mathrm{~K}$ (for $\mathrm{CuAlBe}$ it was less than 2 days at this temperature, and for $\mathrm{CuZnAl}$ it was near 2 hours).

To accelerate the process, temperatures of $388 \mathrm{~K}$ and $398 \mathrm{~K}$ were tested. After near 2 years, the samples seemed to be near a "stationary" transformation temperature (circles and triangles in fig. 3). In order to see if the Rs increase was recoverable, the temperature was lowered to $373 \mathrm{~K}$ (first vertical arrow in figure 3 ). There seemed to be no effect on the transformation temperature for the $398 \mathrm{~K}$-aged sample for near six months. Then, the temperature was increased to $398 \mathrm{~K}$, and the Rs increased slightly for the $388 \mathrm{~K}$-aged sample. For both samples, the transformation temperature does not recover on lowering the temperature at which the sample is kept. Further on, it cannot be ascertained if a really "asymptotic-stationary" state is effectively reached, because the evolution is very slow, within the experimental error of the long time measurements.

The microscopic changes corresponding to the observed transformation temperature evolution might be very small from the structural point of view, and then be very difficult to detect. The reference [17] states that no 
order-disorder transition exists in near-stoichiometric NiTi at elevated temperatures, so fewer contributions from atomic order issues would be expected than in $\mathrm{Cu}$-based alloys. Also, [18] notifies that after aging at $200^{\circ} \mathrm{C}$, the transformation could not be observed in samples of NiTi that, before aging, did transform. On the other hand, [19] reported that no precipitation of Ti3Ni4 was detected in NiTi by TEM even after $3000 \mathrm{~h}$ at $373 \mathrm{~K}$, but precipitation was detectable at $473 \mathrm{~K}$ after $100 \mathrm{~h}$. Then, from the long times implied in our study, it might be that some precipitation phenomena is proceeding in the samples even at temperatures near $373 \mathrm{~K}$, but the changes are structurally very small, yet significant for the transformation temperature changes.

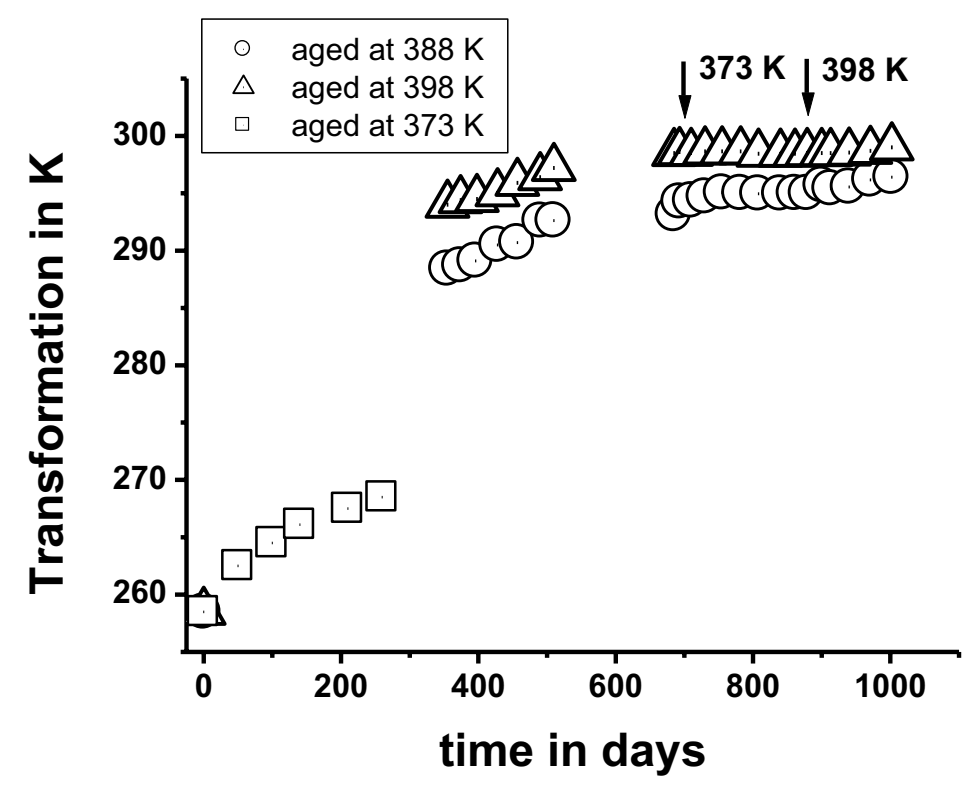

Fig. 3 Transformation temperature (Rs) for NiTi pseudo-elastic wire, as a function of temperature and time at which the samples are kept.

\section{Conclusions}

Some SMAs have diffusion effects at moderate temperatures (near room temperature), giving rise to a transformation temperature evolution related to the time and temperatures at which the sample is kept. $\mathrm{CuZnAl}$ and $\mathrm{CuAlBe}$ alloys show recoverable transformation temperature changes long time after the quench, and a predictive model based in experimental data is able to account for the evolutions, as thermally activated processes, and these effects have been related, in $\mathrm{CuZnAl}$, to atomic ordering.

The $\mathrm{CuAlBe}$ alloy has a time evolution in beta phase much slower than the $\mathrm{CuZnAl}$ alloy, at $100^{\circ} \mathrm{C}$ the time constant for CuAlBe is around 2 days, but only near 2 hours for CuZnAl. The amplitude of the transformation temperature evolution is similar, even though of opposite sign. The transformation temperature of $\mathrm{CuZnAl}$ decreases if the sample is kept at higher temperature after stationary state is reached, but increases if $\mathrm{CuAlBe}$ is kept at higher temperature.

The NiTi alloy has a much slower time evolution that the one for CuAlBe, but it is "non recoverable near room temperature", and the amplitude and time constant are not well determined. This poses a difficulty for long time applications at "moderate" temperatures (near $100^{\circ} \mathrm{C}$ ), but should not be a problem either for short time applications or for applications at lower temperatures (i. e. at $37^{\circ} \mathrm{C}$, a rough estimation of the time constant for the evolution is longer than 100 years)

\section{Acknowledgements}

Support from SMARTeR (ESF-MICINN BIA 2006-27041-E) project, and from C22/06 of 'Ministerio de Fomento' (Spain) is acknowledged. Previous supports for the long-time experiments came from CICYT (Spain). 


\section{References}

[1] K. Otsuka, C.M. Wayman: Shape Memory Materials, Cambridge University Press, Cambridge, U.K., 1998.

[2] M.O. Moroni, R. Saldivia, M. Sarrazin, A. Sepulveda, Mater. Sci. Eng. A 335 (2002) 313-319.

[3] V. Torra, A. Isalgue, F.C. Lovey, F. Martorell, F.J. Molina, M. Sade, H. Tachoire, J. Phys. IV 113 (2004) 8590 .

[4] V. Torra, A. Isalgue, F. Martorell, F.C. Lovey, M. Sade, F.J. Molina:, The 13th World Conference on Earthquake Engineering, Vancouver, Canada, 2004 (published in CD and DVD).

[5] F.C. Lovey, V. Torra: Progr. Mater. Sci. 44 (1999) 189-289.

[6] R. Rapacioli, M. Ahlers: Acta Metall. 27 (1979), p. 777-784

[7] J. Zhang, X. Ren, K. Otsuka, M. Asai: Scripta Mater. 41 (1999), p. 1109-1113

[8] A. Isalgue, V. Torra, F.C. Lovey, J. Therm. Anal. 66 (2001) 7-16.

[9] A. Isalgue and V. Torra. Meas. Sci. Technol. 4 (1993) 456-461.

[10] V. Torra, A. Isalgue, in: M. Koiwa, K. Otsuka, T. Miyazaki (Eds.), Proc. Solid-Solid Phase Transformations PTM'99, The Japan Institute of Metals, 1999, pp. 867-870.

[11] A. Isalgue and V. Torra: J. Phys. IV 7 (1997) pp. C5-339-C5-344.

[12] W. Pfeiler. Acta Metall. 36 (1988), pp. 2417-2434.

[13] A. Isalgue and V. Torra. J. Therm. Anal. 52 (1998), pp. 773-780.

[14] A. Isalgué, V. Torra, J. Fernandez, F.C. Lovey: Materials Science and Engineering A 378 (2004) 227-231

[15] C. Auguet, A. Isalgue, F. C. Lovey, J. L. Pelegrina, S. Ruiz and V. Torra: Journal of Thermal Analysis and Calorimetry, 89 (2007) 2, 537-542.

[16] C. Auguet, A. Isalgue, V. Torra, F. C. Lovey and J. L. Pelegrina: Journal of Thermal Analysis and Calorimetry, 92 (2008) 1, 63-71.

[17] J. Zhang, G. Fan, Y. Zou, X. Ding, X. Ren, J. Sun, K. Nakamura, K. Otsuka: Mat. Sci. Eng. A $438-440$ (2006), p. 608-611

[18] Y. Zheng, F. Jiang, L. Li, H. Yang, Y. Liu: Acta Mater. 56 (2008) p. 736-745

[19] J.I Kim, S. Miyazaki: Acta Mater. 53 (2005) 4545-4554 